\title{
The death of Richard Feynman
}

\section{Richard Feynman was rightly an heroic figure in physics for the best of reasons: he had the flair to make up the rules as he went along. Will young people have heard the message?}

THE death last week of Richard Feynman of the California Institute of Technology is not merely a loss to science but a setback for the principle that science can (and should) be fun. That was the spirit in which his distinctive physics textbook is decorated on the flyleaf with a photograph of him at work on a the bongo drums, but there is much more than that to say.

Feynman's carefully preserved Brooklyn accent allowed him to make even his acerbity amusing, while his pleasure at his many achievements was usually modified for the benefit of observers who might be dazzled by his readiness to cut the figure of a poor boy made good. He was always well-supplied with plausible accounts of how he stumbled into being a genius.

There will be many formal appreciations of Feynman's work in the months ahead. (Nature hopes to publish such a one.) What follows is not that at all, but one view of how Feynman helped to change the way that people think from the early years after the Second World War (Feynman, with others, had been at Los Alamos working on the Manhattan Project) when it became everybody's task to make a sensible quantum theory of electrodynamics, the task from which Dirac had shied away.

There were two difficulties, of which the first was conceptual: whole generations had by then been brought up to understand classical fields such as those of Maxwell's electromagnetism, but what precisely was to be understood by the notion of a field which, like Maxwell's, was not merely a function of place but, to be relativistic, of time as well and, being observable, capable of being represented as an operator, as quantum mechanics requires?

The practitioners in those far-off days did not seriously appreciate what they required of those who listened to them. Perhaps they were forgivably distracted by their second difficulty, that the quantization of the electromagnetic field did not magic away the classical conundrum first made explicit by H.A.Lorentz, that the the energy of the interaction between a charged object and its own field must be infinite. Dirac had had better luck with his theory of the single electron.

The Schwingers and Tomanagas of the times, sharing the general belief that the infinities must be artefacts, hit the problem with all the algebra they could lay their hands upon. It was a triumph of application (not to mention high skill) over great adversity. Self-mockingly simple, Feynman once said he merely wanted properly to understand what the giants had been doing.

One of the reasons why Feynman was always a physicists' physicist is that he really meant that he wanted to understand. The most memorable of the byproducts of that exercise (while still a graduate student, but an exceptional one, at Cornell) are the Feynman diagrams, allpurpose ways of visualizing, classifying and calculating the elements that go into calculating the probability that a system will be transformed into another upon interaction. (Matrix element is the keyword.) Undergraduates who cannot scribble a few Feynman diagrams will not now easily succeed. But that, the prevalence of Feynman diagrams, and the plague of their corruptions, would be a poor monument for a subversive man.

Feynman's enduring achievement was rather that he made the quantum mechanics of fields accessible to others than people skilled at algebra. Starting from a point of view of which Bohr would heartily have approved, he took the view that a quantum system is no different from a classical system in that one thing follows from another. There may be a little local difficulty, not insuperable, in shaking this notion of causality free from that of the expectation that time must be an absolute simulation of some unique clock-face.

What really matters about the evolution of a physical system, Feynman concluded at a tender age, is that it should be possible to calculate the evolving state of any system from a description of its present state in an appropriate language as modified by some operation, mathematical if the language is of that kind. Feynman's propagators (which are the ways of calculating) will ultimately be more important than his diagrams.

More important still is Feynman's way of getting to this state of grace. The textbooks are full of conundrums due to Bohr in the $1920 \mathrm{~s}$ based on his version of the Young's slit gedanken experiment: put an electron source in front of a pair of slits, stop down the output of the source until the electrons are emitted, for practical purposes, singly and then ask why things should be different if one slit is closed.

Feynman appears to have been undismayed by mean gedanken experiments of that kind: "if it's a quantum system, all evolutionary tracks are possible, but it's important to be able to estimate the likelihood of one rather than another". So there emerged Feynman's distinctive formulation of the way that we all now conceive of the deterministic evolution of systems that are no less well-determined for being intrinsically uncertain. That is like pulling two rabbits out of a hat at once. That the infinities melt away is another.

Most of this had been accomplished in the early 1950s. Feynman became perpetually Caltech's most popular lecturer and also (a different business) one of its best teachers, even at such a place. All that was accomplished in about fifteen years.

There are several lessons to be drawn from the Feynman story, of which the chief concerns the nature of the scientific life: there is no cant in the common assertion of great men that they are still learning, even from their students. The name of the game is to understand: to have understood a little better is the prize. But understanding does not require that one should follow the other fellow's algebra in every detail, but that one should look for a language that makes sense. That is what the true subversives practise.

Second, the time quickly comes when it is more economical of genius to teach rather than to practise. It is simply that there is a limit to the energy there can be to spare for the usual competition for space in established journals, and a natural pleasure and evident benefit (if to others) to be won from teaching people in a way that makes them properly subversive also.

Third, there is no end in sight. Feynman's account of quantum mechanics and of the way that quantum systems evolve is in a sense provisional. It may be the best system extant, with applications way outside the quantum electrodynamics for which it was developed, but that does not imply that some young subversive will not one day find an even simpler way of putting the whole message, as Feynman did. It will be a great surprise if one does not. But the need for another simplification is already clamant.

From time to time, candidate successors emerge, but only briefly. Feynman's strength was his physical intuition, his weakness was his flair for being distracted. Einstein, of course, was more persistent, but that was a less distracting age.

John Maddox 